\title{
LET GOLDFINCHES FLY OUT OF YELLOW
}

\author{
wild mustard \\ let the sun rotate as though seeming to circle the earth \\ let rain fall into storm culverts \\ and the blue dragonfly \\ swerve \\ out of blue sky \\ begin to count the beats of your heart \\ then uncount them \\ imitate a tree then imitate \\ a tree imitating you \\ then forget which one \\ you are \\ now arrange crows to reflect sky with their wings \\ and chainsaws \\ to wail across the canyon \\ like lost \\ souls
}

